\title{
Increased crystalloid fluid requirements during zone 3 \\ Resuscitative Endovascular Balloon Occlusion of the Aorta (REBOA) versus Abdominal Aortic and Junctional Tourniquet (AAJT) after class II hemorrhage in swine
}

\author{
Andreas Brännström ${ }^{1}$ (D) - Albin Dahlquist ${ }^{1} \cdot$ Jenny Gustavsson $^{2}$ • Ulf P. Arborelius ${ }^{2} \cdot$ Mattias Günther $^{1}$
}

Received: 13 August 2020 / Accepted: 27 December 2020 / Published online: 30 January 2021

(c) The Author(s) 2021

\begin{abstract}
Purpose Pelvic and lower junctional hemorrhage result in a significant amount of trauma related deaths in military and rural civilian environments. The Abdominal Aortic and Junctional Tourniquet (AAJT) and infra-renal (zone 3) Resuscitative Endovascular Balloon Occlusion of the Aorta (REBOA) are two options for resuscitation of patients with life threatening blood loss from and distal to the pelvis. Evidence suggest differences in the hemodynamic response between AAJT and zone 3 REBOA, but fluid management during resuscitation with the devices has not been fully elucidated. We compared crystalloid fluid requirements (Ringer's acetate) between these devices to maintain a carotid mean arterial pressure (MAP) $>60 \mathrm{mmHg}$. Methods $60 \mathrm{~kg}$ anesthetized and mechanically ventilated male pigs were subjected to a mean 1030 (range 900-1246) $\mathrm{mL}$ ( $25 \%$ of estimated total blood volume, class II) haemorrhage. AAJT $(n=6)$ or zone 3 REBOA $(n=6)$ were then applied for $240 \mathrm{~min}$. Crystalloid fluids were administered to maintain carotid MAP. The animals were monitored for $30 \mathrm{~min}$ after reperfusion.

Results Cumulative resuscitative fluid requirements increased 7.2 times (mean difference $2079 \mathrm{~mL}$; 95\% CI 627-3530 mL) in zone 3 REBOA (mean 2412; range 800-4871 mL) compared to AAJT (mean 333; range 0-1000 mL) to maintain target carotid MAP. Release of the AAJT required vasopressor support with norepinephrine infusion for a mean $9.6 \mathrm{~min}(0.1 \mu \mathrm{g} /$ $\mathrm{kg} / \mathrm{min}$ ), while REBOA release required no vasopressor support.

Conclusion Zone 3 REBOA required 7.2 times more crystalloids to maintain the targeted MAP. The AAJT may therefore be considered in a situation of hemorrhagic shock to limit the need for crystalloid infusions, although removal of the AAJT caused more severe hemodynamic and metabolic effects which required vasopressor support.
\end{abstract}

Keywords Non-compressible hemorrhage $\cdot$ Resuscitation $\cdot$ Aortic tourniquet $\cdot$ REBOA $\cdot$ Prehospital care

\section{Introduction}

Trauma is a major global health issue contributing to about $10 \%$ of overall mortality and an annual worldwide death of more than 5.8 million people [1, 2]. Hemorrhage is still the leading cause of potentially preventable death among trauma

Andreas Brännström

andreas.brannstrom@ki.se

1 Department of Clinical Science and Education, Södersjukhuset, Karolinska Institutet, Sjukhusbacken 10, S1, SE-118 83 Stockholm, Sweden

2 Department of Neuroscience, Karolinska Institutet, Stockholm, Sweden victims [2, 3]. Non-compressible hemorrhage from the abdomen, pelvis, junctional regions and proximal lower extremities constitutes a particular challenge since exsanguination can occur rapidly and conventional interventions are ineffective [4]. In the military setting, $19 \%$ of haemorrhage-related deaths 2001-2011 occurred from the junctional regions only, emphasizing an injury pattern of growing importance in armed conflicts [5-7].

Resuscitative endovascular balloon occlusion of the aorta (REBOA) and the Abdominal Aortic and Junctional Tourniquet (AAJT) are devices with the potential to control hemorrhage from the lower body including the pelvis by closing off arterial inflow [8-10]. Occlusion of the aorta may also benefit resuscitation by providing 
proximal hemodynamic support and reduce the requirement of resuscitative fluids. The AAJT is designed for external compression of the abdominal aorta resulting in infra-renal (zone 3) occlusion while the endovascular REBOA balloon can be positioned at any level within the aorta. Zone 3 REBOA and the AAJT are equally effective in achieving hemostasis [11]. For patients with traumatic hemorrhage, current guidelines in the US recommend a zone 3 location of the REBOA balloon for isolated injuries including and distal to the pelvis [12]. Both devices constitute potential prehospital and battlefield interventions to decrease mortality from non-compressible torso hemorrhage. In conflict environments, the AAJT may be the only realistic option to stabilize and evacuate a casualty with massive non-compressible hemorrhage below the aortic bifurcation [13]. One major benefit of the noninvasive AAJT is the ease of use compared to the REBOA technique which requires percutaneous or surgical femoral arterial access. However, the REBOA balloon may be a more suitable option in higher levels of care with the possibility to maintain hemostasis during surgery and adaptable reperfusion under vigorous hemodynamic monitoring. We have shown that a transition from the AAJT to zone 3 REBOA may be safely performed with hemodynamic support, in a report where we suggested hemodynamic differences between the interventions [14]. We have also shown that reduction of the perfused vascular volume by the AAJT reduced crystalloid fluid requirements to maintain vital circulation compared to fluid resuscitation only [8]. Although causing less severe ischemia and being better tolerated after reperfusion, zone 3 REBOA has limited resuscitative hemodynamic effects compared to a zone 1 location [15].

A recent animal study described improved blood pressure support when resuscitating with whole blood and fresh frozen plasma compared to crystalloids when comparing the AAJT and REBOA in a pooled group. The AAJT and REBOA animals did not differ in hemodynamics, metabolic responses or survival [16]. In contrast, our experience suggests that the AAJT and zone 3 REBOA lead to different hemodynamic situations, and that REBOA requires increased fluid resuscitation to maintain blood pressure.

A large volume of crystalloids is an independent factor for increased morbidity and mortality in trauma patients. Fluid resuscitation of patients with impending or manifest hemorrhagic shock should replace sufficient blood volume to maintain cardiac output and end-organ perfusion while preserving blood coagulation and oxygenation. Crystalloids address the first of these parameters while having a negative impact on coagulation and oxygen transportation $[17,18]$. No comparison has been reported on crystalloid fluid management during zone 3 REBOA and the AAJT. $\mathrm{We}$, therefore, compared crystalloid fluid requirements to maintain carotid MAP $>60 \mathrm{mmHg}$ during a $4 \mathrm{~h}$ prolonged application of the AAJT and zone 3 REBOA after a class II (25\%) hemorrhage.

\section{Materials and methods}

This study was approved and conducted in accordance with the Swedish regional ethics approval board for animal research (S3-15), and included four phases: animal preparation, hemorrhage, intervention (AAJT or zone 3 REBOA) and reperfusion (Fig. 1).

\section{Surgical preparation and instrumentation}

Castrated, crossbred male pigs (54-65 kg) were pre-medicated with $150 \mathrm{mg}$ tiletamine/zolazepam (Zoletil $100 \mathrm{Vet}$ ) and $6 \mathrm{mg}$ medetomidine (Domitor) and placed supine on a standard operating table. $100 \% \mathrm{O}_{2}$ was administered via a nose cone for three minutes before anesthesia induction through the right auricular vein with pentobarbital $6 \mathrm{mg} /$ $\mathrm{kg}$, atropine $0.02 \mathrm{mg} / \mathrm{kg}$ and $2.5 \mu \mathrm{g} / \mathrm{kg}$ fentanyl. Animals were orally intubated with an $8 \mathrm{~mm}$ endotracheal tube and mechanical ventilation (Hamilton $\mathrm{C} 2$ ventilator, Hamilton medical, Geneva Switzerland) was adjusted to $\mathrm{PaCO}_{2}$ $4.7-5.5 \mathrm{kPa}$. Initial ventilator settings were: inspiratory oxygen fraction $21 \%$; peak inspiratory pressure $16 \mathrm{~cm} \mathrm{H}_{2} \mathrm{O}$ and peak expiratory end pressure $4 \mathrm{~cm} \mathrm{H}_{2} \mathrm{O}$. Anesthesia

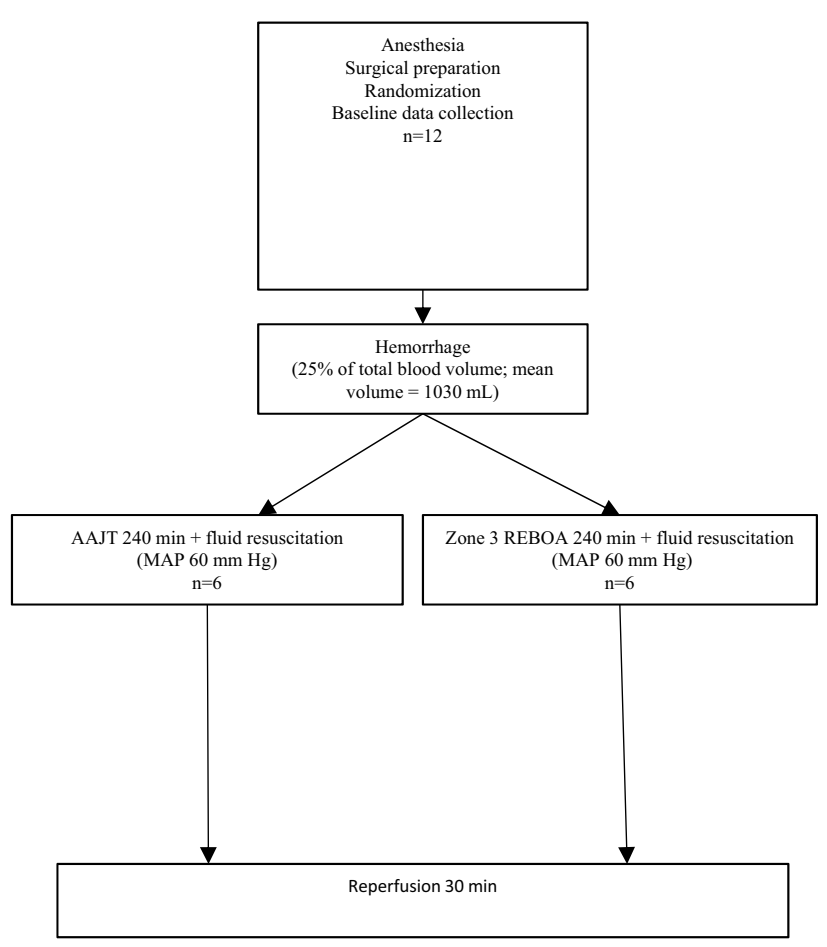

Figure 1 Experimental design 
was maintained by continuous infusion of ketamine $25 \mathrm{mg} /$ $\mathrm{kg} / \mathrm{h}$ (Ketaminol Vet $100 \mathrm{mg} / \mathrm{ml}$ ), midazolam 0, $0485 \mathrm{mg} /$ $\mathrm{kg} / \mathrm{h}$ (Midazolam Hameln $1 \mathrm{mg} / \mathrm{ml}$ ) and fentanyl $3.5 \mu \mathrm{g} /$ $\mathrm{kg} / \mathrm{h}$ (Fentanyl B. Braun $50 \mu \mathrm{g} / \mathrm{ml}$ ). A fluid bolus of $500 \mathrm{ml}$ Ringer's acetate was given after induction of anesthesia to adjust baseline fluid-balance discrepancies and then continuously throughout the experiments with $3 \mathrm{ml} / \mathrm{kg} / \mathrm{h}$ to replace insensible losses.

A 7.5 F Swan-Ganz pulmonary artery catheter (Edwards Lifescience) was introduced in the surgically exposed right external jugular vein and used for core temperature, cardiac output (CO) and mixed venous oxygen saturation $\left(\mathrm{SvO}_{2}\right)$. The left carotid artery was surgically exposed and used for hemorrhage, hemodynamic monitoring and collection of blood samples. A suprapubic urine catheter and 12 EKG electrodes were placed to monitor heart rate, the occurrence of arrhythmias and to verify euthanasia. The AAJT was prepositioned and left in place unbuckled. In the REBOA animals, a 7F introducer (REBOA Medical, Båstad, Norway) was placed via the femoral artery using Seldinger-technique.

\section{Induction of hemorrhage shock}

A $25 \%$ of estimated total blood volume hemorrhage, modified from an earlier study with hemorrhage in two phases under hemodynamic monitoring, was utilized to create a reproducible state of class II hemorrhage [19]. TBV was estimated by $67 \mathrm{ml} / \mathrm{kg}$. Initially $50 \%$ of calculated blood loss was drawn for 7 min by a peristaltic pump (Masterflex $\mathrm{L} / \mathrm{S}$, Cole Parmer) and $450 \mathrm{ml}$ of shed blood was stored in citrated bags at $38^{\circ} \mathrm{C}$. After a 10 min stabilization of MAP, as a response to splenic autotransfusion, hemorrhage was continued at half the rate until target volume was achieved.

\section{Interventions}

Animals were assigned to groups AAJT application (AAJT; $n=6$ ) or zone 3 REBOA (REBOA; $n=6$ ). After completion of hemorrhage the AAJT was inflated $(300 \mathrm{mmHg}$ ) or a $15 \mathrm{~mm}$ balloon (REBOA Medical, Båstad Norway) was positioned in the infra-renal aorta guided by anatomical measurement on the skin from the site of vascular access to aortic bifurcation before insertion and inflated with $8 \mathrm{ml} \mathrm{NaCl}$. We have verified anatomical measurement as a robust method of estimating the level of the balloon in our earlier experimental series [14]. A zone 3 location of the balloon was verified in all animals postmortem via laparotomy. $\mathrm{T}_{0}$ was defined as when the AAJT or REBOA balloon was fully inflated. Complete data collection started at $\mathrm{T}_{0}$. Total occlusion of aortic blood flow was verified by loss of a pulse wave and no blood flow in a percutaneous arterial catheter placed distally to the devices, non-measurable noninvasive blood pressure on the hind leg, non-measurable heart rate (HR) and pulse-oximetry from the tail probe.

\section{Intravenous fluid resuscitation}

In accordance with current Tactical Combat Casualty Care battlefield resuscitation guidelines [20] for situations with no access to whole blood, component therapy or colloids, Ringer's acetate was administered in $250 \mathrm{ml}$ bolus infusions as needed, following AAJT or REBOA inflation, if $\mathrm{MAP}<60 \mathrm{mmHg}$. At $240 \mathrm{~min}$ the animals were transfused with $450 \mathrm{ml}$ autologous blood from stored citrated bags until MAP approximated baseline. The AAJT or the REBOA balloon was then promptly deflated. Additional rapid crystalloid infusion of $2000 \mathrm{~mL}$ Ringer's acetate was continued throughout the reperfusion phase in all animals. Norepinephrine $(0.1 \mu \mathrm{g} / \mathrm{kg} / \mathrm{min})$ was infused if MAP $<60 \mathrm{mmHg}$. After $30 \mathrm{~min}$, animals were euthanized by $40 \mathrm{ml}$ pentobarbital sodium (Alfatal Vet, $100 \mathrm{mg} / \mathrm{ml}$ ).

\section{Data-collection and statistical analyses}

Hemodynamic performance and fluid administration were continuously registered and arterial blood gases and hemoglobin were collected. Arterial pressure was measured between 0-200 $\mathrm{mm} \mathrm{Hg}$ and monitored as maximum (systolic), mean (automatically calculated) and minimum (diastolic). The following calculations were used for hemodynamic parameters: Stroke volume $(\mathrm{SV})=\mathrm{CO} / \mathrm{HR}, \mathrm{SVR}=80 \times(\mathrm{MAP}-\mathrm{CVP}) /$ CO [21]. Primary outcome was cumulative crystalloid fluid requirements to maintain MAP $>60 \mathrm{mmHg}$. Secondary outcomes were hemodynamic and metabolic $(\mathrm{pH}$, base excess, lactate, hematocrit and core body temperature) parameters. A power calculation for a continuous outcome superiority trial required 6 animals per group to have an $80 \%$ chance of detecting a $2500 \mathrm{ml}$ difference in the primary outcome $(\alpha=0.05)$. Standard deviations were calculated from pilot studies. For five animals in the AAJT group, a secondary analysis of previously published data was performed [8] and one animal was added according to the power calculation.

All statistical analyses were made by GraphPad Prism version 7.03 for Windows (GraphPad Software). $p<0.05$ was considered significant. All data are expressed as mean \pm SD. Two-way ANOVAs with multiple comparisons tests were used for iv fluids, hemorrhage volume, hemodynamic- and metabolic parameters. Student's unpaired t-test was used for norepinephrine. 
Table 1 Baseline characteristics and hemodynamic parameters

\begin{tabular}{llll}
\hline & AAJT $_{240}$ & REBOA $_{240}$ & $p$ value \\
\hline Body weight $(\mathrm{kg})$ & 57.5 & 61.5 & $\mathrm{~ns}^{*}$ \\
Hemorrhage (mL) & $980(168)$ & $1080(102)$ & 0.25 \\
Systolic blood pressure (mmHg) & $139(15)$ & $143(10)$ & $>0.99$ \\
Mean arterial pressure (mmHg) & $115(16)$ & $103(14)$ & 0.93 \\
Heart rate (/min) & $105(22)$ & $112(34)$ & $>0.99$ \\
Cardiac output (L/min) & $6.4(1.5)$ & $6.15(2.4)$ & $>0.99$ \\
Stroke volume (mL) & $61(6)$ & $56(17)$ & 0.998 \\
Systemic vascular resistance & $1453(415)$ & $1509(614)$ & $>0.99$ \\
$\quad($ dynes*sec*cm & & & \\
\hline
\end{tabular}

Group means (SD), *not significant

\section{Results}

Baseline characteristics displayed differences for MAP which was higher in the AAJT group and heart rate which was higher in the REBOA group (Table 1). Average blood loss was $980 \mathrm{ml}$ (AAJT) and $1080 \mathrm{ml}$ (REBOA) (Table 1). The hemorrhage protocol resulted in a MAP reduction of $50 \%$ (AAJT) and 70\% (REBOA), respectively (Fig. 3). Interventions with AAJT or REBOA were performed according to the protocol with no complications or malfunctions of the devices (Fig. 2). The application time did not exceed one minute in both study groups.

All animals survived the intervention and subsequent 30 min reperfusion phase. Both interventions caused a MAP increase of more than $100 \%$ (Fig. 3). The systolic blood pressure increased by $67 \%$ (AAJT) and $122 \%$ (REBOA), respectively (Fig. 3). Peak MAP and SBP occurred at $\mathrm{T}_{20}$ in the AAJT and $\mathrm{T}_{40}$ in the REBOA group (Fig. 3). After deflation of the AAJT and REBOA balloon, an expected decrease in MAP and SBP occurred within one minute. After norepinephrine infusion was started, MAP was kept $>60$ and $\mathrm{SBP}>90 \mathrm{mmHg}$. Average cumulative resuscitative fluids at 240 min were $2412 \mathrm{ml}$ (range: $800-4800 \mathrm{~mL}$ ) in the REBOA group and $333 \mathrm{ml}$ (range: $0-1000 \mathrm{~mL}$ ) in the AAJT group (Fig. 3). Mean difference in resuscitative fluids was $2079 \mathrm{~mL}$ (95\% CI 627-3530 mL). Four animals resuscitated with the AAJT upheld a MAP $>60 \mathrm{mmHg}$ without crystalloid fluids. Systemic vascular resistance (SVR) increased after both interventions but was significantly higher in the AAJT group between $\mathrm{T}_{0}$ and $\mathrm{T}_{30}$. After $\mathrm{T}_{60} \mathrm{SVR}$ had little variation until reperfusion when it decreased, with no trend of stabilization (Fig. 3). Release of the AAJT required vasopressor support with norepinephrine infusion for a mean time of $9.6 \mathrm{~min}$ and a mean total of $55.2 \mu \mathrm{g}(0.1 \mu \mathrm{g} / \mathrm{kg} / \mathrm{min})$, while REBOA animals required no vasopressor support. Cardiac output displayed no differences between interventions and showed a little increase after AAJT/REBOA balloon inflation (Fig. 4). After deflation of the AAJT/REBOA balloon
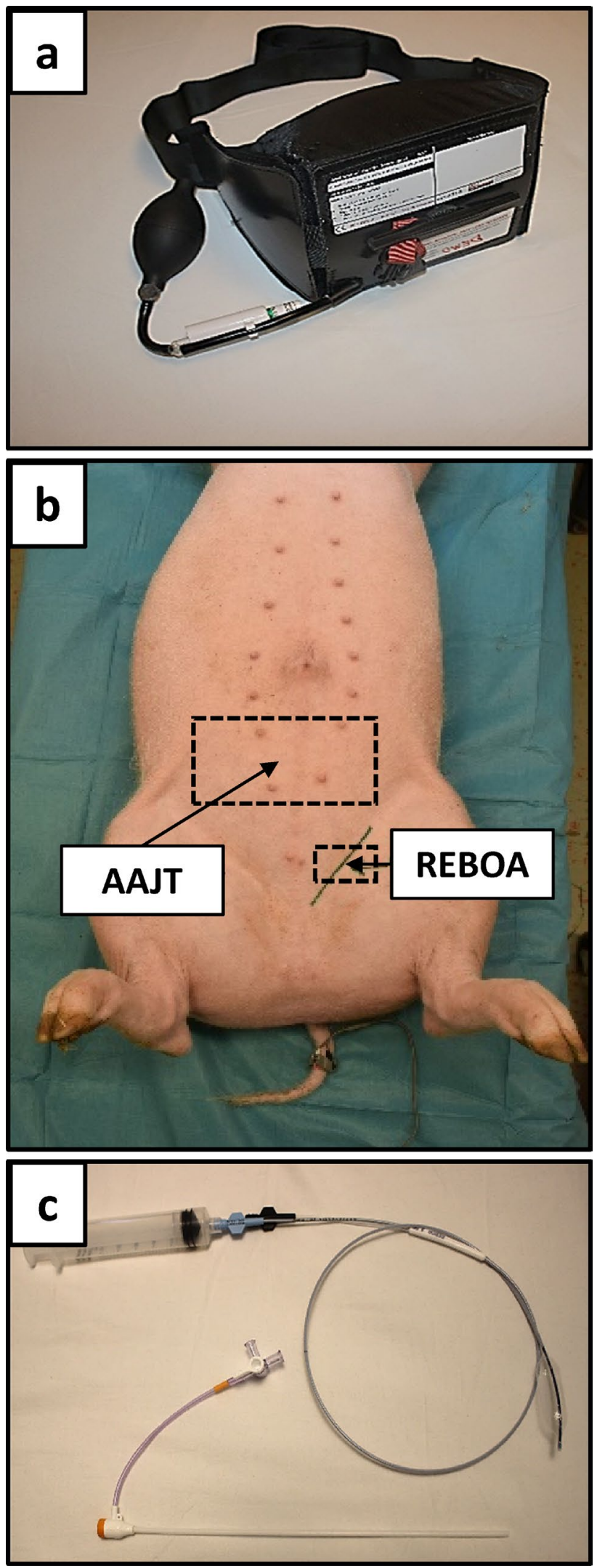

Figure 2 Abdominal view of a $60 \mathrm{~kg}$ laboratory animal b with anatomic landmarks for aortic occlusion devices; AAJT a and REBOA balloon catheter with $7.5 \mathrm{~F}$ introducer $\mathbf{c}$ 

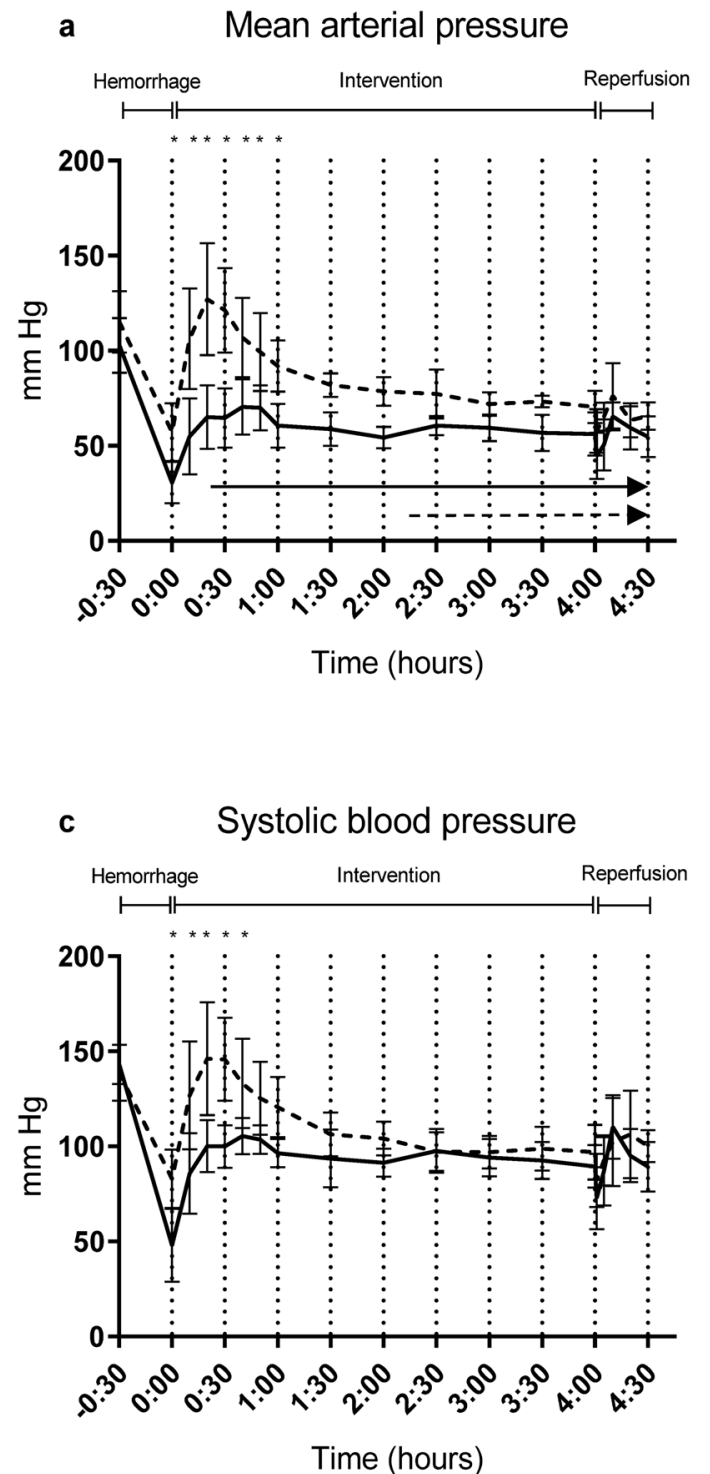

\section{b Systemic vascular resistance}

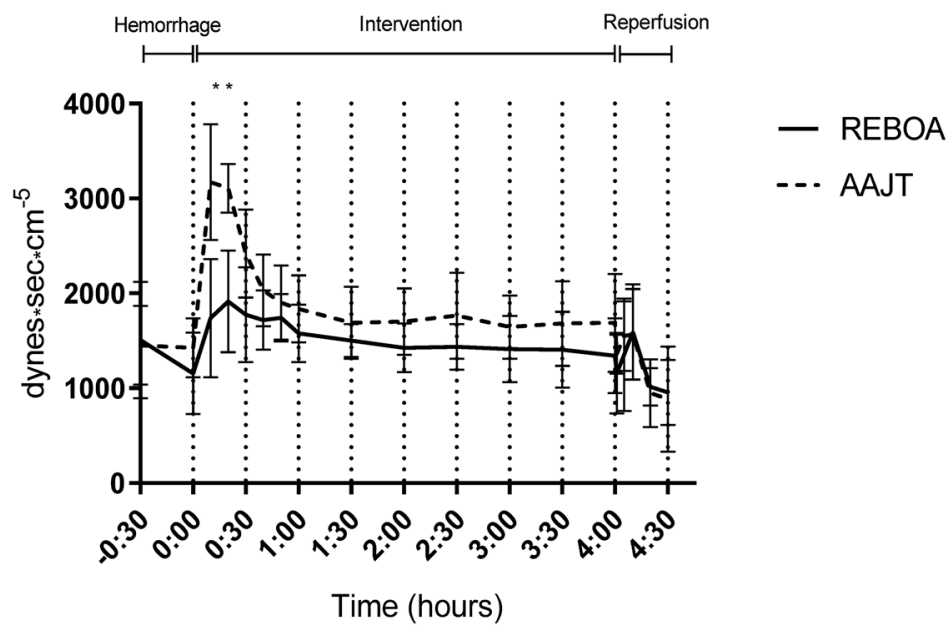

d $\quad$ Resuscitative fluids

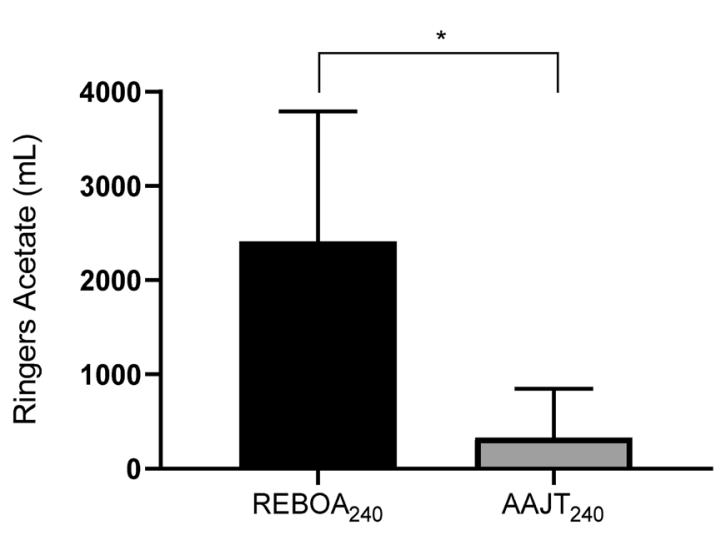

Figure 3 Hemodynamic changes during experiments a-c and cumulative crystalloid infusions between groups d. Arrows in a represents crystalloid infusion times. Data are presented as mean with standard deviation. A $p<0.05$ was considered significant. * $p<0.05$

cardiac output increased to supra-normal in the AAJT animals. Heart rates increased after AAJT application throughout the experiment with near $100 \%$ increase compared to REBOA (Fig. 4). After reperfusion heart rates increased further for five (AAJT) and ten (REBOA) minutes respectively. Stroke volume was significantly higher in the REBOA group during the intervention phase (Fig. 4). After deflation, SV increased simultaneously in both study groups after five (AAJT) and ten (REBOA) minutes. No signs of ventricular fibrillation or prolonged Q-T interval appeared on EKG during the experiments.

Hematocrit increased in the AAJT group compared to REBOA. AAJT application caused an increase of hematocrit by $17 \%$ within the first $30 \mathrm{~min}$, whereafter it decreased towards baseline (Fig. 5). REBOA application caused a decrease in hematocrit by $27 \%$ after hemorrhage, until autologous transfusion and reperfusion were performed. Lactate showed a slight and simultaneous increase in both groups after hemorrhage until $\mathrm{T}_{90}$ with a responding decrease in base excess (Fig. 5). Between $\mathrm{T}_{150}$ and end of experiment, lactate was significantly higher in the AAJT animals. Both interventions caused a sharp increase in lactate after deflation. Core body temperature increased in AAJT animals compared to REBOA animals which in contrast were hypothermic (Fig. 5). Deflation of the devices caused an immediate decrease in temperature in both groups with no trend towards normalization. 

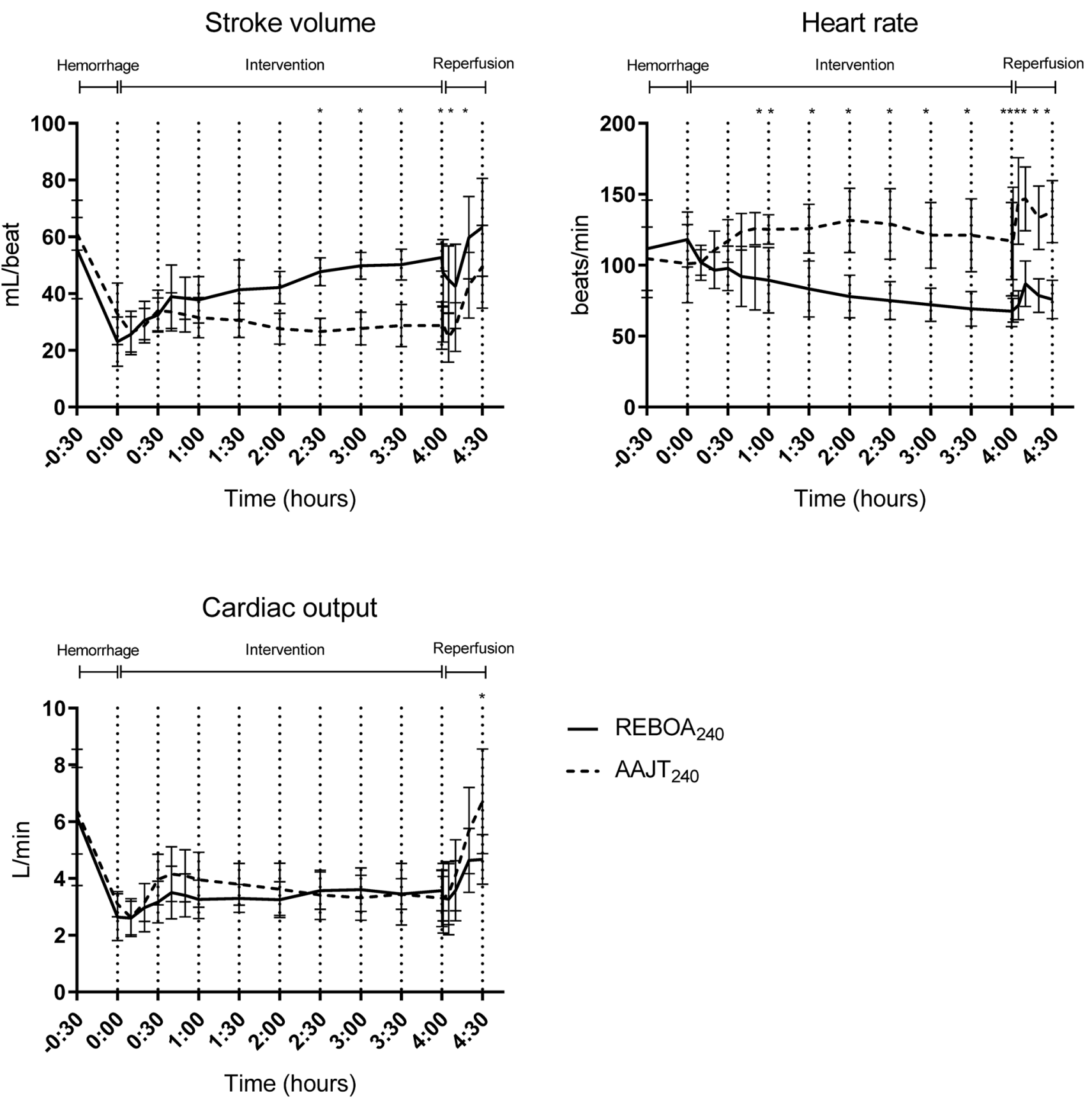

Figure 4 Cardiac performance between groups during experiments. Data are presented as mean with standard deviation. A $p<0.05$ was considered significant. $* p<0.05$

\section{Discussion}

In this study we demonstrate differences in crystalloid fluid requirements between zone $3 \mathrm{REBOA}$ and the AAJT to maintain a target MAP. It is possible that a zone 3 REBOA offers limited hemodynamic support without blood volume replacement in hemorrhagic shock, compared to AAJT, which may affect the applicability of the devices in different clinical situations.
Based on the effect on hemodilution and hypothermia which we observed when comparing AAJT with crystalloid fluid resuscitation only [8], we aimed to demonstrate a $2500 \mathrm{~mL}$ difference in crystalloid fluids between the devices. In remote battlefield or civilian prehospital environments this amount may represent a near exhaustion of available resuscitative fluids. Although the study did not reach $2500 \mathrm{~mL}$ assumed in the power analysis, the difference of $2079 \mathrm{~mL}$ suggests clinically important differences 

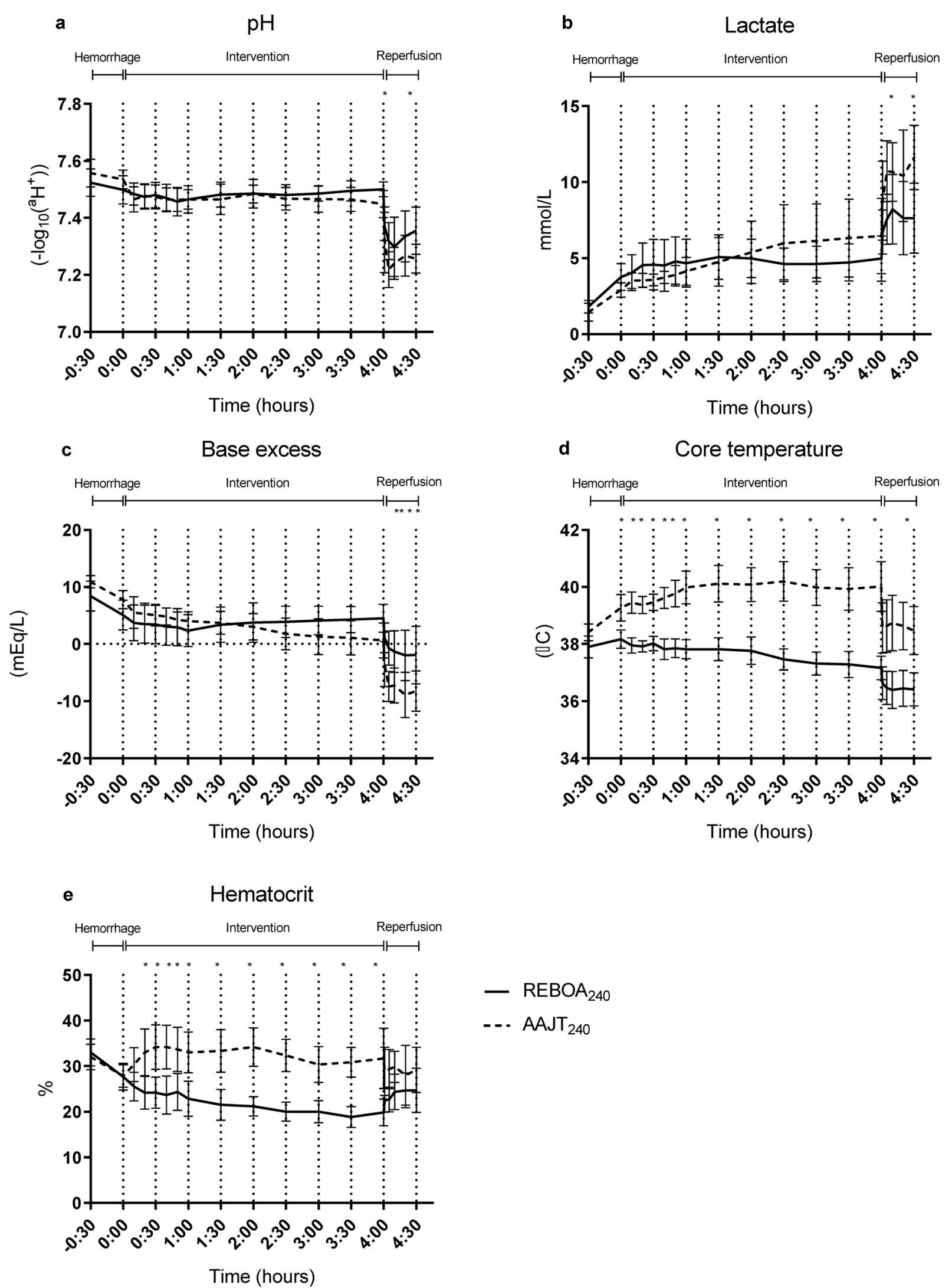

Figure 5 Multipanel figure of metabolic changes during experiments. Data are presented as mean with standard deviation. A $p<0.05$ was considered significant. $*=p<0.05$ 
in crystalloid fluid management between the interventions. Importantly, only two animals in the AAJT group required fluid resuscitation in addition to the device application to maintain vital perfusion.

The differences in hemodynamics between the devices are likely explained by an interruption of splanchnic circulation by the AAJT with a subsequent increased systemic vascular resistance. Furthermore, compression of the vena cava and reduced cardiac preload likely explains the elevated heart rates and reduced stroke volumes in the AAJT animals although both groups had similar cardiac output. While our findings contrast a study by Rall et al. where no significant hemodynamic differences were reported except for carotid MAP [11], Tibbits et al. compared hemodynamic effects of zone 1 to zone 3 REBOA and also reported limited hemodynamic support from zone 3 REBOA [15]. The AAJT increases the intra-abdominal pressure and therefore higher ventilator pressures may be expected during mechanical ventilation. The hemodynamic impact of increased intrathoracic pressures during positive pressure ventilation should be addressed in future studies.

A complete separation of blood flow between tissues proximal and distal to the AAJT likely caused more severe ischemic insults after reperfusion, with an accumulation of anaerobic metabolites causing increased metabolic acidosis after device removal. Vasopressor support after AAJT removal was not required after zone 3 REBOA, and suggested a more severe ischemic injury in the AAJT group. The increased amount of crystalloid fluids to maintain MAP above $60 \mathrm{mmHg}$ in the REBOA animals likely explained the difference in hematocrit between groups. It is possible that REBOA animals where diluted while AAJT animals had extravascular plasma shift from the increase in blood pressure. In addition, the significantly lower core body temperature in the REBOA animals may have caused coagulopathy. Future studies addressing fluid resuscitation in conjunction with aortic occlusion devices should include analyses of coagulation. We found no significant differences in the metabolic parameters $\mathrm{pH}$, lactate and base excess during the intervention which implied adequate resuscitation. Worth noting is the differing composition of Ringer's lactate and Ringer's acetate, the latter principally used in Scandinavia. The theoretical advantage of acetate as a buffer is the systemic metabolism compared to the liver-dependent lactate metabolism. Large volumes of Ringer's lactate have been associated with increased lactate levels compared to acetate solutions while not affecting the $\mathrm{pH}$ [22], which is why it is likely that Ringer's acetate did not cause any significant changes in lactate levels in the present study.

From a prehospital point of view, possible complications associated with REBOA must be emphasised. The tolerance of the device is limited by ischemia. In addition; arterial access, catheter insertion and balloon positioning risk vascular injury, unsuccessful resuscitation and vital organ ischemia [23]. Furthermore, the correct location of the balloon is crucial but may be hard to determine, and the possibility to monitor the physiology in austere environments may be challenging [24]. REBOA may therefore not be a feasible option in battlefield conditions, compared to the AAJT.

Current guidelines limit AAJT application to 60 min to avoid permanent ischemic injuries [25-27]. We applied a prolonged application time to investigate crystalloid fluid requirements for $240 \mathrm{~min}$. The application time was chosen by the authors based on their experience and a possible scenario of delayed definite treatment after major bleeding in an emergency scenario. Robust preclinical hemodynamic data may guide fluid resuscitation in such an event. Prolonged application may occur in complex environments such as in battlefields which raises practical and ethical considerations. A successful prehospital resuscitation and evacuation of a patient with the AAJT may also warrant transition to zone 3 REBOA during surgical treatment which constitutes another rationale for investigating the fluid requirements beyond $60 \mathrm{~min}$. Kheirabadi et al. demonstrated permanent ischemic injuries to the spinal cord after AAJT application exceeding $60 \mathrm{~min}$. Spinal cord injuries have not been studied after prolonged REBOA [12, 25] why studies addressing safe time limits for zone 3 REBOA are needed. An important finding of the present study were increased $\mathrm{pH}$ and base excess and lower lactate after prolonged zone 3 REBOA, suggesting that the AAJT should be replaced with zone 3 REBOA when feasible, to avoid deteriorating ischemic effects.

The study has some limitations to be discussed. The swine model is frequently used in translational research on physiological response to hemorrhage and interventions, but the translation to humans should be done with caution. Another limitation is the method of haemorrhage by a controlled bleeding from the carotid artery. We did not perform measurements on coagulation, $\mathrm{BE}$ and $\mathrm{pH}$ on collected blood before auto-transfusion. The coagulation system may be activated differently in traumatic haemorrhage, which should be addressed in future studies. Citrate may affect the electrolyte- and metabolic balance in patients, especially after rapid and massive transfusions. However, impaired citrate metabolism in humans is mainly associated with liver disease, and administration of citrated blood was limited to one bag of $450 \mathrm{~mL}$ in animals with normal liver function. No hypocalcaemia was detected. Therefore, the influence of citrated blood transfusion likely had a negligible impact on the metabolic results. The study design allowed for comparisons of fluid resuscitation with a limited number of experimental animals, in accordance of the principle of reduction of experimental animals, from the principles of the 3Rs (Replacement, Reduction and Refinement), the framework for ethical animal research. However, as a consequence, the short observation time 
after reperfusion was also a major limitation. A longer intensive care phase would allow for assessments of organ function and general physiologic consequences including inflammation, coagulation and renal failure. The increased vasopressor need after removal of the AAJT is likely caused by redistribution of blood from the ischemic vasculature distal to the AAJT together with a reperfusion injury with ensuing inflammatory response and capillary leakage. It is unclear how and to what extent these mechanisms contribute, and an assessment would require a longer follow-up, which would also allow for investigations of postoperative fluid management. Future studies should therefore include a longer reperfusion phase, covering the first $24 \mathrm{~h}$.

We did not assess central venous pressure (CVP). To correctly assess the central venous blood volume indicated by CVP, the intra-thoracic pressure from positive pressure ventilation should have been equal between groups. The AAJT applies pressure towards the diaphragm causing higher ventilator pressures to maintain adequate tidal volumes and gas exchange in the lungs, which is why CVP likely was related to ventilatory pressures. In this study, the animals were mechanically ventilated to maintain et $\mathrm{CO}_{2}$ within a normal range, and adjustments in ventilatory pressures were made accordingly. Future studies may investigate CVP in relation to the devices in spontaneous ventilation. Finally, the positive pressure mechanical ventilation was also a limitation since the effect on cardiac preload possibly differs between the interventions. Future studies may compare the impact of positive pressure ventilation and spontaneous ventilation when applying AAJT and zone 3 REBOA.

\section{Conclusion}

Zone 3 REBOA required 7.2 times more crystalloids to maintain the targeted MAP. The AAJT may therefore be considered in a situation of hemorrhagic shock to limit the need for crystalloid infusions, although removal of the AAJT caused more severe hemodynamic and metabolic effects which required vasopressor support.

Acknowledgements We would like to thank Lars-Gunnar Olsson for excellent technical assistance.

Author contributions AB: literature review, study design, data collection, data analysis, data interpretation, manuscript writing and revision. AD: data collection. JG: data collection, manuscript revision. UA: data collection, manuscript revision. MG: literature review, study design, data collection, data analysis, data interpretation, manuscript writing and revision.

Funding Open Access funding provided by Karolinska Institute. This study was funded by the Swedish armed forces.

\section{Compliance with ethical standards}

Conflict of interest Andreas Brännström, Albin Dahlquist, Jenny Gustavsson, Ulf Arborelius and Mattias Günther declare that they have no conflict of interest.

Ethics approval This study was approved and conducted in accordance with the Swedish regional ethics approval board for animal research (S3-15).

Open Access This article is licensed under a Creative Commons Attribution 4.0 International License, which permits use, sharing, adaptation, distribution and reproduction in any medium or format, as long as you give appropriate credit to the original author(s) and the source, provide a link to the Creative Commons licence, and indicate if changes were made. The images or other third party material in this article are included in the article's Creative Commons licence, unless indicated otherwise in a credit line to the material. If material is not included in the article's Creative Commons licence and your intended use is not permitted by statutory regulation or exceeds the permitted use, you will need to obtain permission directly from the copyright holder. To view a copy of this licence, visit http://creativecommons.org/licenses/by/4.0/.

\section{References}

1. Roth GA, Abate D, Abate KH, Abay SM, Abbafati C, Abbasi N, Abbastabar H, Abd-Allah F, Abdela J, et al. Global, regional, and national age-sex-specific mortality for 282 causes of death in 195 countries and territories, 1980-2017: a systematic analysis for the Global Burden of Disease Study 2017. Lancet. 2018;392(10159):1736-88.

2. Spahn DR, Bouillon B, Cerny V, Duranteau J, Filipescu D, Hunt BJ, Komadina R, Maegele M, Nardi G, Riddez L, et al. The European guideline on management of major bleeding and coagulopathy following trauma: fifth edition. Crit Care. 2019;23(1):98.

3. Caspers M, Maegele M, Frohlich M. Current strategies for hemostatic control in acute trauma hemorrhage and trauma-induced coagulopathy. Expert Rev Hematol. 2018;11(12):987-95.

4. Alarhayem AQ, Myers JG, Dent D, Liao L, Muir M, Mueller D, Nicholson S, Cestero R, Johnson MC, Stewart R, et al. Time is the enemy: mortality in trauma patients with hemorrhage from torso injury occurs long before the "golden hour." Am J Surg. 2016;212(6):1101-5.

5. Eastridge BJ, Mabry RL, Seguin P, Cantrell J, Tops T, Uribe P, Mallett O, Zubko T, Oetjen-Gerdes L, Rasmussen TE, et al. Death on the battlefield (2001-2011): implications for the future of combat casualty care. J Trauma Acute Care Surg. 2012;73(6 Suppl 5):S431-7.

6. Walker NM, Eardley W, Clasper JC. UK combat-related pelvic junctional vascular injuries 2008-2011: implications for future intervention. Injury. 2014;45(10):1585-9.

7. Stannard A, Morrison JJ, Scott DJ, Ivatury RA, Ross JD, Rasmussen TE. The epidemiology of noncompressible torso hemorrhage in the wars in Iraq and Afghanistan. J Trauma Acute Care Surg. 2013;74(3):830-4.

8. Brannstrom A, Rocksen D, Hartman J, Nyman N, JG BS, Arborelius UP, Gunther M, . Abdominal Aortic and Junctional Tourniquet release after $240 \mathrm{~min}$ is survivable and associated with small intestine and liver ischemia after porcine class II hemorrhage. J Trauma Acute Care Surg. 2018;85(4):717-24. 
9. Stannard A, Eliason JL, Rasmussen TE. Resuscitative endovascular balloon occlusion of the aorta (REBOA) as an adjunct for hemorrhagic shock. J Trauma. 2011;71(6):1869-72.

10. Morrison JJ, Lendrum RA, Jansen JO. Resuscitative endovascular balloon occlusion of the aorta (REBOA): a bridge to definitive haemorrhage control for trauma patients in Scotland? Surgeon. 2014;12(3):119-20.

11. Rall JM, Redman TT, Ross EM, Morrison JJ, Maddry JK. Comparison of zone 3 Resuscitative Endovascular Balloon Occlusion of the Aorta and the Abdominal Aortic and Junctional Tourniquet in a model of junctional hemorrhage in swine. J Surg Res. 2018;226:31-9.

12. Bulger EM, Perina DG, Qasim Z, Beldowicz B, Brenner M, Guyette F, Rowe D, Kang CS, Gurney J, DuBose J, et al. Clinical use of resuscitative endovascular balloon occlusion of the aorta (REBOA) in civilian trauma systems in the USA, 2019: a joint statement from the American College of Surgeons Committee on Trauma, the American College of Emergency Physicians, the National Association of Emergency Medical Services Physicians and the National Association of Emergency Medical Technicians. Trauma Surg Acute Care Open. 2019;4(1):e000376.

13. Handford C, Parker PJ. The Potential use of the Abdominal Aortic Junctional Tourniquet( $\mathrm{R})$ in a military population: a review of requirement, effectiveness, and usability. J Spec Oper Med. 2019;19(4):74-9.

14. Brannstrom A, Dahlquist A, Gustavsson J, Arborelius UP, Gunther M. Transition from abdominal aortic and junctional tourniquet to zone 3 resuscitative endovascular balloon occlusion of the aorta is feasible with hemodynamic support after porcine class IV hemorrhage. J Trauma Acute Care Surg. 2019;87(4):849-55.

15. Tibbits EM, Hoareau GL, Simon MA, Davidson AJ, DeSoucy ES, Faulconer ER, DuBose JJ, Neff LP, Grayson JK, Williams TK, et al. Location is everything: the hemodynamic effects of REBOA in zone 1 versus zone 3 of the aorta. J Trauma Acute Care Surg. 2018;85(1):101-7.

16. Schechtman DW, Kauvar DS, De Guzman R, Polykratis IA, Prince MD, Kheirabadi BS, Dubick MA. Differing resuscitation with aortic occlusion in a swine junctional hemorrhage polytrauma model. J Surg Res. 2019;248:90-7.

17. Jones DG, Nantais J, Rezende-Neto JB, Yazdani S, Vegas P, Rizoli S. Crystalloid resuscitation in trauma patients: deleterious effect of 5L or more in the first 24h. BMC Surg. 2018;18(1):93.
18. Jenkins DH, Rappold JF, Badloe JF, Berseus O, Blackbourne L, Brohi KH, Butler FK, Cap AP, Cohen MJ, Davenport R, et al. Trauma hemostasis and oxygenation research position paper on remote damage control resuscitation: definitions, current practice, and knowledge gaps. Shock. 2014;41(Suppl 1):3-12.

19. Markov NP, Percival TJ, Morrison JJ, Ross JD, Scott DJ, Spencer JR, Rasmussen TE. Physiologic tolerance of descending thoracic aortic balloon occlusion in a swine model of hemorrhagic shock. Surgery. 2013;153(6):848-56.

20. Butler FK, Holcomb JB, Schreiber MA, Kotwal RS, Jenkins DA, Champion HR, Bowling F, Cap AP, Dubose JJ, Dorlac WC, et al. Fluid resuscitation for hemorrhagic shock in Tactical Combat Casualty Care: TCCC Guidelines change 14-01-2 June 2014. J Spec Oper Med. 2014;14(3):13-38.

21. Drobin D, Gryth D, Persson JK, Rocksen D, Arborelius UP, Olsson LG, Bursell J, Kjellstrom BT. Electroencephalogram, circulation, and lung function after high-velocity behind armor blunt trauma. J Trauma. 2007;63(2):405-13.

22. Rajan S, Srikumar S, Tosh P, Kumar L. Effect of lactate versus acetate-based intravenous fluids on acid-base balance in patients undergoing free flap reconstructive surgeries. J Anaesthesiol Clin Pharmacol. 2017;33(4):514-9.

23. Davidson AJ, Russo RM, Reva VA, Brenner ML, Moore LJ, Ball C, Bulger E, DuBose JJ, Moore EE, Rasmussen TE, et al. The pitfalls of REBOA: risk factors and mitigation strategies. J Trauma Acute Care Surg. 2017;84(1):192-202.

24. Chaudery M, Clark J, Morrison JJ, Wilson MH, Bew D, Darzi A. Can contrast-enhanced ultrasonography improve Zone III REBOA placement for prehospital care? J Trauma Acute Care Surg. 2016;80(1):89-94.

25. Kheirabadi BS, Terrazas IB, Miranda N, Voelker AN, Klemcke HG, Brown AW, Dubick MA. Long-term consequences of abdominal aortic and junctional tourniquet for hemorrhage control. J Surg Res. 2018;231:99-108.

26. Kheirabadi BS, Dubick MA. Safe duration of Abdominal Aortic and Junctional Tourniquet application. J Trauma Acute Care Surg. 2019;87(3):740-1.

27. Gunther M, Brannstrom A. Reply to letter: Kheirabadi et al. Long-term consequences of abdominal aortic and junctional tourniquet for hemorrhage control. J Trauma Acute Care Surg. 2019;87(3):741. 